International Journal of Modern Physics A

(C) World Scientific Publishing Company

DPNU-05-17

September, 2005

\title{
Vector Manifestation and the Hidden Local Symmetry*
}

\author{
Masayasu Harada \\ Department of Physics, Nagoya University, Nagoya 464-8602, Japan $^{\dagger}$
}

\begin{abstract}
In this write-up, I summarize the key ingredients of the vector manifestaion formulated in the hidden local symmetry theory, in which the $\rho$ meson becomes massless degenerated with the pion at the chiral phase transition point.

Keywords: Chiral Symmetry Restoration, Vector Meson
\end{abstract}

\section{Introduction}

The vector manifestation (VM) was proposed $\frac{112}{2}$ as a novel manifestation of Wigner realization of chiral symmetry where the vector meson $\rho$ becomes massless at the chiral phase transition point. Accordingly, the (longitudinal) $\rho$ becomes the chiral partner of the Nambu-Goldstone boson $\pi$. The VM provides a strong support for Brown-Rho scaling 3 which predicted that the mass of light-quark hadrons should drop in proportion to the quark condensate $\langle\bar{q} q\rangle$. Actually, the dilepton spectra observed in the KEK-PS E325 4 and the CBELSA/TAPS $[5]$ as well as in the CERN/SPS 6 can be explained by the dropping mass of $\rho / \omega$ based on the Brown-Rho scaling 7 .

In this write-up, I first briefly review the difference between the VM and the conventional manifestation of chiral symmetry restoration based on the linear sigma model in section 2. Then, I show how to formulate the VM in hot matter in section 3. Finally, in section 4 I give a brief summary.

\section{Vector Manifestation of Chiral Symmetry}

Let me discuss the difference between the VM and the conventional manifestation of chiral symmetry restoration based on the linear sigma model, the GL manifestation, in terms of the chiral representation of the low-lying mesons by extending the analyses done in Ref. 8 for two flavor QCD.

The VM is characterized by

$$
(\mathrm{VM}) \quad f_{\pi}^{2} \rightarrow 0, \quad m_{\rho}^{2} \rightarrow m_{\pi}^{2}=0, \quad f_{\rho}^{2} / f_{\pi}^{2} \rightarrow 1,
$$

*Talk given at "International Conference on QCD and Hadronic Physics" (June 16-20, 2005, Beijing, China).

${ }^{\dagger}$ Electronic address : harada@hken.phys.nagoya-u.ac.jp 
where $f_{\rho}$ is the decay constant of (longitudinal) $\rho$ at $\rho$ on-shell. This is completely different from the GL manifestation where the scalar meson $S$ becomes massless degenerate with $\pi$ as the chiral partner

I first consider the representations of the following zero helicity $(\lambda=0)$ states under $\mathrm{SU}(3)_{\mathrm{L}} \times \mathrm{SU}(3)_{\mathrm{R}}$; the $\pi$, the (longitudinal) $\rho$, the $S$ and the (longitudinal) axial-vector meson denoted by $A_{1}$. The $\pi$ and the $A_{1}$ are admixture of $(8,1) \oplus(1,8)$ and $\left(3,3^{*}\right) \oplus\left(3^{*}, 3\right)$ since the symmetry is spontaneously broken 8 .

$$
\begin{aligned}
|\pi\rangle & =\left|\left(3,3^{*}\right) \oplus\left(3^{*}, 3\right)\right\rangle \sin \psi+|(8,1) \oplus(1,8)\rangle \cos \psi, \\
\left|A_{1}(\lambda=0)\right\rangle & =\left|\left(3,3^{*}\right) \oplus\left(3^{*}, 3\right)\right\rangle \cos \psi-|(8,1) \oplus(1,8)\rangle \sin \psi,
\end{aligned}
$$

where the experimental value of the mixing angle $\psi$ is given by approximately $\psi=\pi / 4$ [ The $\rho$ and the $S$, on the other hand, are expressed as

$$
|\rho(\lambda=0)\rangle=|(8,1) \oplus(1,8)\rangle, \quad|S\rangle=\left|\left(3,3^{*}\right) \oplus\left(3^{*}, 3\right)\right\rangle .
$$

When the chiral symmetry is restored at the phase transition point, it is natural to expect that the chiral representations coincide with the mass eigenstates: The representation mixing is dissolved. From Eq. (2) one can easily see that there are two ways to express the representations in the Wigner phase of the chiral symmetry: The conventional GL manifestation corresponds to the limit $\psi \rightarrow \pi / 2$ :

$$
\left\{\begin{aligned}
|\pi\rangle,|S\rangle & \rightarrow\left|\left(3,3^{*}\right) \oplus\left(3^{*}, 3\right)\right\rangle, \\
|\rho(\lambda=0)\rangle,\left|A_{1}(\lambda=0)\right\rangle & \rightarrow|(8,1) \oplus(1,8)\rangle .
\end{aligned}\right.
$$

On the other hand, the VM corresponds to the limit $\psi \rightarrow 0$ in which the $A_{1}$ goes to a pure $\left(3,3^{*}\right) \oplus\left(3^{*}, 3\right)$, now degenerate with the scalar meson $S$ in the same representation, but not with $\rho$ in $(8,1) \oplus(1,8)$ :

$$
(\mathrm{VM}) \quad\left\{\begin{aligned}
|\pi\rangle,|\rho(\lambda=0)\rangle & \rightarrow|(8,1) \oplus(1,8)\rangle \\
\left|A_{1}(\lambda=0)\right\rangle,|s\rangle & \rightarrow\left|\left(3,3^{*}\right) \oplus\left(3^{*}, 3\right)\right\rangle .
\end{aligned}\right.
$$

Namely, the degenerate massless $\pi$ and (longitudinal) $\rho$ at the phase transition point are the chiral partners in the representation of $(8,1) \oplus(1,8)$.

\section{Formulation of the Vector Manifestation in Hot Matter}

It should be noticed that the critical temperature of the chiral symmetry restoration is approached from the broken phase up to $T_{c}-\epsilon$, and that the following basic assumptions are adopted in the analysis: (1) The relevant degrees of freedom until near $T_{c}-\epsilon$ are only $\pi$ and $\rho$; (2) Other mesons such as $A_{1}$ and scalar mesons are still heavy at $T_{c}-\epsilon$; (3) Partial chiral symmetry restoration already occurs at $T_{c}-\epsilon$. Based on these assumptions, it was shown 91011 that the VM necessarily occurs at the chiral symmetry restoration point.

One of the most important key ingredients to formulate the VM in hot matter is the intrinsic temperature dependences 9 of the parameters determined through the Wilsonian matching 12. The intrinsic temperature dependence introduced through 
the Wilsonian matching is nothing but the signature that hadrons have internal structures constructed from the quarks and gluons. This is similar to the situation where coupling constants among hadrons are replaced with appropriate momentumdependent form factors in high energy region. The intrinsic effects play more important roles in higher temperature region, especially near the critical temperature.

When $T_{c}$ is approached from below, the axial-vector and vector current correlators derived in the OPE approach each other for any value of $Q^{2}$. Thus we require that these current correlators in the HLS become close to each other near $T_{c}$ for any value of $Q^{2}$ around $\Lambda^{2}$. This requirement implies that the bare $g$ and $a$ satisfy

$$
g_{\text {bare }}(T) \underset{T \rightarrow T_{c}}{\longrightarrow} 0, \quad a_{\text {bare }}(T) \underset{T \rightarrow T_{c}}{\longrightarrow} 1 .
$$

These conditions ("VM conditions in hot matter") for the bare parameters are converted into the conditions for the on-shell parameters through the Wilsonian renormalization group equations (RGEs). Since $g=0$ and $a=1$ are separately the fixed points of the RGEs for $g$ and $a \underline{13},(g, a)=(0,1)$ is satisfied at any energy scale. As a result, the quantum correction to the $\rho$ mass as well as the hadronic thermal correction disappears at $T_{c}$ since they are proportional to the gauge coupling $g$. The bare $\rho$ mass, which is also proportional to $g_{\text {bare }}$, vanishes at $T_{c}$. These imply that the pole mass of the $\rho$ meson also vanishes at $T_{c}$ :

$$
m_{\rho}^{2}(T) \rightarrow 0 \text { for } T \rightarrow T_{c} .
$$

\section{Summary}

In this write-up, I first showed the difference between the VM and the conventional manifestation of chiral symmetry restoration based on the linear sigma model in terms of the chiral representation of the mesons in section 2 Then, in section 3 I reviewed how to formulate the VM in hot matter. I would like to note that the VM in dense matter can be formulated in a similar way 14 , where the intrinsic density dependence plays an important role.

There are several predictions of the VM in hot matter made so far: In Ref. 15, the vector and axial-vector susceptibilities were studied. It was shown that the equality between two susceptibilities are satisfied and that the VM predicts $\chi_{A}=$ $\chi_{V}=\frac{2}{3} T_{c}^{2}$ for $N_{f}=2$, which is in good agreement with the result obtained in the lattice simulation 16]. In Ref. 10, a prediction associated with the validity of vector dominance (VD) in hot matter was made: As a consequence of including the intrinsic effect, the VD is largely violated at the critical temperature. The violation of the VD near the critical temperature plays an important role to explain the NA60 dimuon data based on the Brown-Rho scaling 17. In Ref. 18, the pion velocity $v_{\pi}$ was studied including the effect of Lorentz symmetry breaking. The prediction for $v_{\pi}$ is $v_{\pi}\left(T_{c}\right)=0.83-0.99$, which is consistent with $v_{\pi}(T)=0.65$ of Cramer et al. 19 extracted from the recent STAR data 20]. Furthermore, in Ref. 21] starting with an HLS Lagrangian at the VM fixed point that incorporates the heavy-quark symmetry and matching the bare theory to QCD, we calculated the splitting of 
chiral doublers of $D$ mesons proposed in Refs. 22. The predicted splitting comes out to be $0.31 \pm 0.12 \mathrm{GeV}$, which is in good agreement with the experiment 23 .

\section{Acknowledgements}

I would like to thank Doctor Youngman Kim, Professor Mannque Rho, Doctor Chihiro Sasaki and Professor Koichi Yamawaki for collaboration in several works done for the vector manifestaion on which this talk is based. This work is supported in part by the JSPS Grant-in-Aid for Scientific Research (c) (2) 16540241, the Daiko Foundataion \#9099, and the 21st Century COE Program of Nagoya University provided by Japan Society for the Promotion of Science (15COEG01).

\section{References}

1. M. Harada and K. Yamawaki, Phys. Rev. Lett. 86, 757 (2001).

2. M. Harada and K. Yamawaki, Phys. Rept. 381, 1 (2003).

3. G. E. Brown and M. Rho, Phys. Rev. Lett. 66, 2720 (1991); Phys. Rept. 396, 1 (2004).

4. K. Ozawa et al. [E325 Collaboration], Phys. Rev. Lett. 86, 5019 (2001); M. Naruki et al., arXiv:nucl-ex/0504016

5. D. Trnka et al. [CBELSA/TAPS Collaboration], Phys. Rev. Lett. 94, 192303 (2005).

6. G. Agakishiev et al. [CERES Collaboration], Phys. Rev. Lett. 75, 1272 (1995).

7. See, e.g., G. Q. Li, C. M. Ko and G. E. Brown, Phys. Rev. Lett. 75, 4007 (1995); R. Rapp and J. Wambach, Adv. Nucl. Phys. 25, 1 (2000).

8. F. J. Gilman and H. Harari, Phys. Rev. 165, 1803 (1968); S. Weinberg, Phys. Rev. 177, 2604 (1969).

9. M. Harada and C. Sasaki, Phys. Lett. B 537, 280 (2002).

10. M. Harada and C. Sasaki, Nucl. Phys. A 736, 300 (2004).

11. For a review of the VM in hot matter, see, e.g., C. Sasaki, Doctral thesis (Nagoya University) "Chiral phase transition in QCD and vector manifestation," arXiv:hep-ph/0504073.

12. M. Harada and K. Yamawaki, Phys. Rev. D 64, 014023 (2001).

13. M. Harada and K. Yamawaki, Phys. Rev. Lett. 83, 3374 (1999).

14. M. Harada, Y. Kim and M. Rho, Phys. Rev. D 66, 016003 (2002).

15. M. Harada, Y. Kim, M. Rho and C. Sasaki, Nucl. Phys. A 727, 437 (2003).

16. C. R. Allton et al., Phys. Rev. D 66, 074507 (2002).

17. G. E. Brown and M. Rho, arXiv:nucl-th/0509001 arXiv:nucl-th/0509002

18. C. Sasaki, Nucl. Phys. A 739, 151 (2004); M. Harada, Y. Kim, M. Rho and C. Sasaki, Nucl. Phys. A 730, 379 (2004).

19. J. G. Cramer, G. A. Miller, J. M. S. Wu and J. H. S. Yoon, Phys. Rev. Lett. 94, 102302 (2005).

20. J. Adams et al. [STAR Collaboration], Phys. Rev. Lett. 92, 112301 (2004); Phys. Rev. C 71, 044906 (2005).

21. M. Harada, M. Rho and C. Sasaki, Phys. Rev. D 70, 074002 (2004).

22. M. A. Nowak, M. Rho and I. Zahed, Phys. Rev. D 48, 4370 (1993); Acta Phys. Polon. B 35, 2377 (2004);

W. A. Bardeen and C. T. Hill, Phys. Rev. D 49, 409 (1994).

23. B. Aubert et al. [BABAR Collaboration], Phys. Rev. Lett. 90, 242001 (2003);

D. Besson et al. [CLEO Collaboration], Phys. Rev. D 68, 032002 (2003);

P. Krokovny et al. [Belle Collaboration], Phys. Rev. Lett. 91, 262002 (2003). 